\title{
Supporting Information for: Confined DART-MS for rapid chemical analysis of electronic cigarette aerosols and spiked drugs
}

\author{
Thomas P. Forbes ${ }^{1, *}$ and Shannon T. Krauss ${ }^{2, *}$ \\ ${ }^{1}$ National Institute of Standards and Technology, Materials Measurement Science Division, \\ Gaithersburg, MD 20899, USA \\ ${ }^{2}$ RTI International, Research Triangle Park, NC 27709, USA \\ *Corresponding authors: TPF: thomas.forbes@nist.gov; STK: skrauss@rti.org
}

\section{Supporting Information Table of Contents}

\section{Supplemental Discussion}

MS Response: Coil Power

\section{Supplemental Figures}

Figure S1. MS response as a function of increasing coil power.

Figure S2. Illustrative spectra and chronograms of select e-liquids and spiked drugs. 


\section{MS Response: Coil Power}

Figure S1(a) displays the signal intensity for THC as a function of increasing coil power for ECIG 2 (ECIG 3 exhibited similar results). The overall signal intensity (i.e., peak area) monotonically increased as the coil power and therefore temperature increased. The observed behavior followed documented trends, specifically three regimes of atomization. ${ }^{1}$ These included 1) powers less than approximately $30 \mathrm{~W}$ that had insufficient power and heating for stable atomization, 2) powers in the range of $50 \mathrm{~W}$ to $95 \mathrm{~W}$ that demonstrated near linear response (signal intensity correlated to aerosolization), and 3) powers above $95 \mathrm{~W}$ that led to over-heating and potential charring of the wick. Interestingly, the demonstrated increase in signal with power also coincided with a sharper, almost step change, increase in observed phthalate peaks for di-n-butyl phthalate and di(2ethylhexyl) phthalate (Figure S1(b)). No coil-related metals (e.g., chromium, nickel, manganese, cadmium, or lead) were readily observed in the tests conducted here. This was potentially because no metals were present in the measured aerosols or metals were present but not detected due to insufficient ionization energy (i.e., metals remain neutral) or system sensitivity. 


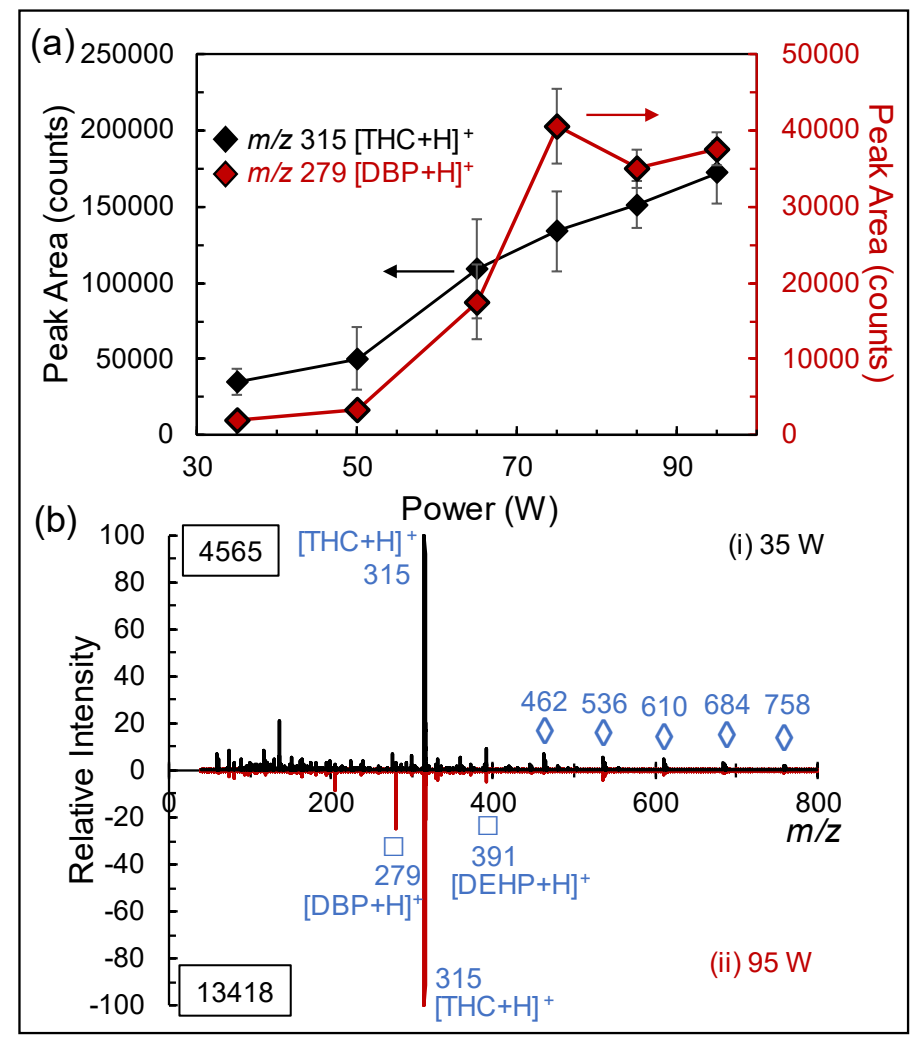

Figure S1. (a) Peak area of THC and di-n-butyl phthalate (DBP) as a function of coil power for 5 $\mu \mathrm{g} / \mathrm{mL}$ THC spiked in an aqueous liquid from ECIG 2. Data points and uncertainty represent the average and standard deviation of 10 replicate measurements. Arrows indicate appropriate axis: black diamonds for THC on left y-axis and red diamonds for DBP on right y-axis. (b) Representative mass spectra for (i) $35 \mathrm{~W}$ and (ii) $95 \mathrm{~W}$ coil power settings. Base peak absolute intensity noted in insets for each spectrum. Preliminary peak assignments can be found in Table 1. 


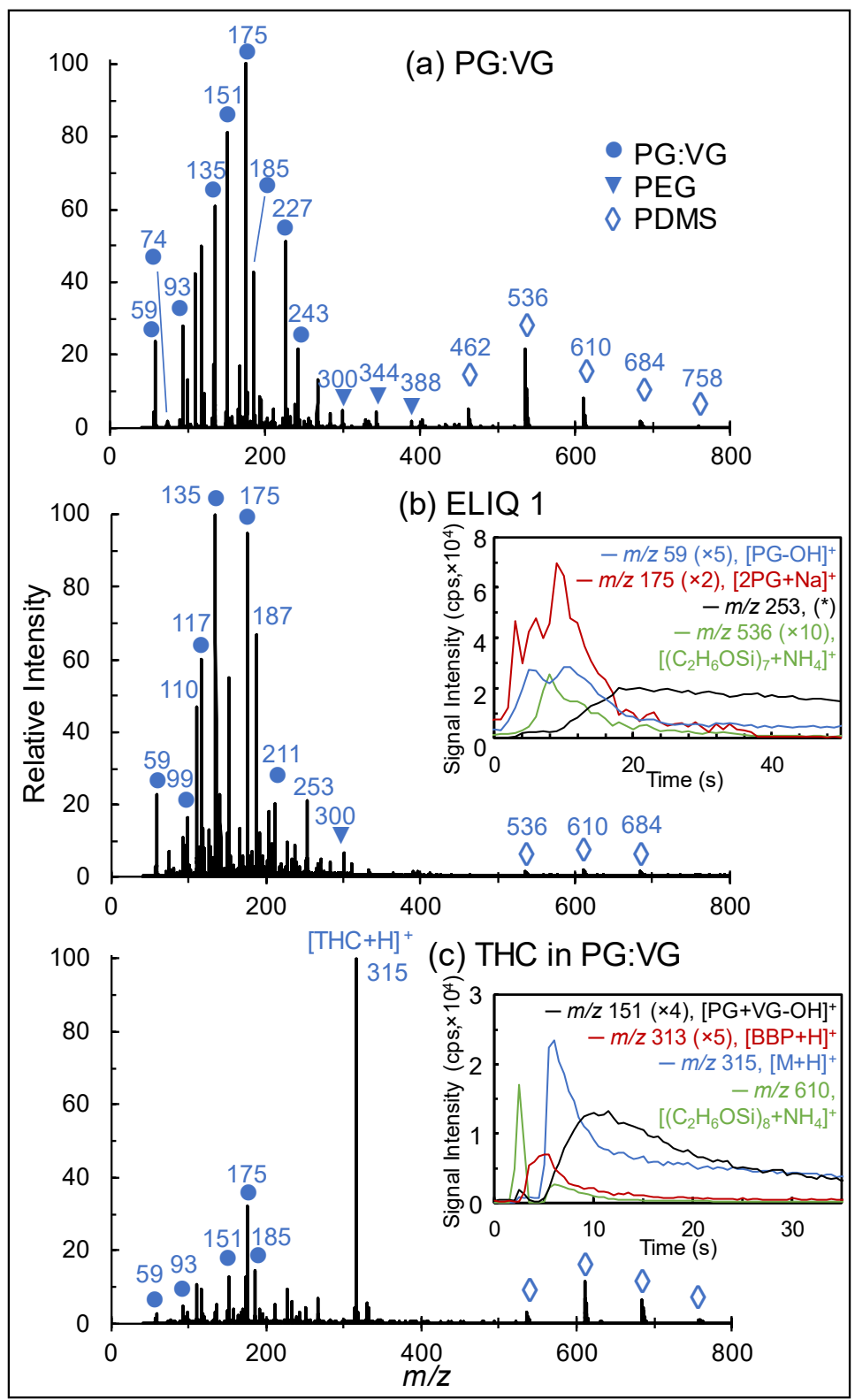

Figure S2. Representative mass spectra for (a) blank PG:VG e-liquid, (b) ELIQ 1 e-liquid, and (c) $250 \mu \mathrm{g} / \mathrm{mL}$ THC spiked in PG:VG e-liquid. Insets display extracted ion chronograms of select species. Preliminary peak assignments can be found in Table 1.

\section{References}

1. Soulet, S.; Duquesne, M.; Toutain, J.; Pairaud, C.; Lalo, H., Influence of Coil Power Ranges on the E-Liquid Consumption in Vaping Devices. International Journal of Environmental Research and Public Health 2018, 15 (9), 1853. 